\title{
Hypertension does not alter disturbances in leptin signalling observed in experimental model of tauopathy
}

\author{
Martin Cente ${ }^{1,2}$, Tomas Smolek ${ }^{1,3}$, Stefan Zorad ${ }^{4}$, Lubica Fialova ${ }^{1,2}$, Natalia Paulenka Ivanovova ${ }^{2}$, \\ Katarina Krskova ${ }^{4}$, Lucia Balazova ${ }^{4}$, Rostislav Skrabana ${ }^{1,2}$ and Peter Filipcik ${ }^{1,2}$ (D) \\ ${ }^{1}$ Institute of Neuroimmunology, Slovak Academy of Sciences, Bratislava, Slovakia \\ ${ }^{2}$ Axon Neuroscience R\&D Services SE, Bratislava, Slovakia \\ ${ }^{3}$ Neuroimmunology institute n.p.o., Bratislava, Slovakia \\ ${ }^{4}$ Institute of Experimental Endocrinology, Biomedical Research Center, Slovak Academy of Sciences, Bratislava, Slovakia
}

\begin{abstract}
Neurodegeneration is associated with hypertension and disturbance in fat metabolism. The complex interaction of neurodegenerative processes with both metabolic changes and blood pressure is still not fully elucidated. Here we demonstrate that the experimentally induced tauopathy in hypertensive transgenic animals causes significant downregulation of plasma leptin (53\% of control), reduction of body weight by $11 \%$, a 1.2-fold drop of adiposity index, and decrease in HDL cholesterol level, while the fasting glucose and insulin concentration remain unchanged. Despite of these alterations we found the leptin projection circuit including the arcuate nucleus, paraventricular nucleus in hypothalamus, and nucleus tractus solitarius in the brainstem not affected by neurofibrillary pathology. Furthermore, hypertension does not alter disturbances in leptin signalling. The presented data provide further insight into neurodegeneration-induced metabolic alterations relevant for human tauopathies.
\end{abstract}

Key words: Neurodegeneration - Tau - Leptin - Hypertension - Transgenic rat

\begin{abstract}
Abbreviations: AD, Alzheimer's disease; ARC, arcuate nucleus; $\mathrm{DMH}$, dorsomedial hypothalamic nucleus; IPGTT, intraperitoneal glucose tolerance test; NTS, nucleus tractus solitarius; PVH, paraventricular hypothalamic nucleus; SHR, spontaneously hypertensive rat; TG, transgenic animals; VMH, ventromedial hypothalamic nucleus; WKY, Wistar Kyoto (rat strain); WT, wild type.
\end{abstract}

\section{Introduction}

Neurodegenerative diseases represent a serious problem in the current human population. Important factors associated with neurodegenerative pathology and its consequences are metabolic disturbances including body weight alterations and disordered fat metabolism (Lee 2011; McGuire and Ishii 2016). Hypertension is another wide-spread health threatening condition associated with development of neurodegeneration. The

Correspondence to: Peter Filipcik, Institute of Neuroimmunology, Slovak Academy of Sciences, Dubravska cesta 9, 84510 Bratislava, Slovakia

E-mail: peter.filipcik@savba.sk molecular mechanisms of these alterations in context with neuronal degeneration are not well understood. It is because the neurodegeneration in humans can start and progress silently and unobserved, a long time before the first symptoms of cognitive or neurological impairment become phenotypically evident. Recently it was published that satiety hormone leptin can contribute to neurogenesis and attenuates neurodegeneration in animal model (Calio et al. 2021). Epidemiologic observations in humans indicate the paradoxical association of body mass index with dementia: it may represent either risk factor of neurodegeneration in persons with overweight in middle life or protective effect in later life (Emmerzaal et al. 2015). Moreover, it was found that leptin can reduce the inflammation and oxidative stress in the cardiomyocytes under

(c) The Authors 2021. This is an open access article under the terms of the Creative Commons Attribution-NonCommercial 4.0 International License (https://creativecommons.org/licenses/by-nc/4.0/), which permits non-commercial use, distribution, and reproduction in any medium, provided the original work is properly cited. 
hypoxia, the pathological events closely associated also with the progress of neurodegenerative pathology (Abd Alkhaleq et al. 2020). Some other studies on adipokines including leptin indicate association of their levels with blood pressure in human (Pantsulaia et al. 2009; Wang et al. 2012). It was shown that elevated plasma leptin correlates with blood pressure of hypertensive patients, however, this association is independent of body adiposity both in normotensive and in hypertensive individuals (Beltowski 2006; Simonds et al. 2017).

Taking together, data from literature suggest that low levels of leptin could accelerate neurodegeneration in both normotensive and hypertensive conditions.

Previously we have demonstrated a significant down-regulation of peripheral leptin in normotensive Wistar Kyoto rat strain with tauopathy (WKY72). Decline in peripheral level of leptin was found as a direct consequence of tauopathy induced by transgenic expression of truncated tau protein, which was designed according to the pathological isoforms found in the brain of Alzheimer's disease (AD) sufferers (Cente et al. 2020).

The aim of this study was to determine the effect of experimentally-induced tauopathy on peripheral metabolic parameters in animals suffering from spontaneous hypertension. Specifically, we aimed to measure the levels of leptin and investigate whether or not the peripheral alterations can be a consequence of the neurofibrillary pathology potentially present in the leptin projection circuit such as the arcuate nucleus, paraventricular nucleus in the hypothalamus, and nucleus tractus solitarius in the brainstem.

\section{Materials and Methods}

\section{Animals}

Spontaneously hypertensive rats (SHR) with chronically elevated systolic blood pressure used in this study were characterized elsewhere (Stozicka et al. 2010). Transgenic rat model of tauopathy (SHR72) was generated by pronuclear injection of truncated tau gene construct into 1-day-old SHR embryos, which were implanted into pseudopregnant females. The expression of truncated human tau protein (aa151-391,4R) is under the control of the brain specific mouse Thy- 1 promoter and is therefore restricted to the central nervous system, brain and upper part of spinal cord. The onset of expression starts early after birth as published before (Zilka et al. 2006; Koson et al. 2008; Stozicka et al. 2010). Rats were housed in standard laboratory conditions in plastic cages $(555 \times 345 \times 195 \mathrm{~mm})$ in a temperature and humidity-controlled environment with a 12/12-hour light/dark cycle (light phase: 7 a.m.-19 p.m.) and with food (Altromin Spezialfutter, Germany, 1324 Maintenance diet for rats and mice, metabolized energy $3.226 \mathrm{cal} / \mathrm{g}$ ) and water available ad libitum. Efforts were made to minimize the suffering and number of experimental animals. Animal cohort comprised of 6 month old transgenic SHR72 rat males (TG, $n=10$ ) and age-matched SHR wild type littermates (WT, $n=10$ ). Animals were assessed randomly. During experiments and analysis, the investigators were blinded to genotype and experimental group. Animal experiments were performed between 9 and 11 a.m. of the light phase. Rats were anesthetized by intraperitoneal injection of a cocktail containing Zoletil 100 (30 mg/kg) (Virbac S.A, Carros, France) and Xylariem (10 mg/kg) (Ecuphar N.V, Oostkamp, Belgium) and then euthanized by decapitation.

\section{Immunohistochemistry}

Animals for immunohistochemistry were perfused with phosphate bovine serum (PBS). The brains were postfixed in $4 \%$ paraformaldehyde for $24 \mathrm{~h}$, embedded in paraffin and cut on a microtome (Leica RM2255) providing sagittal serial section $(8 \mu \mathrm{m})$. For staining of brain areas (brainstem (BS), nucleus tractus solitarius (NTS), arcuate nucleus (ARC), ventromedial nucleus (VMH), dorsomedial nucleus (DMH) and paraventricular nucleus (PVH)), the sections between coordinates $0.4 \mathrm{~mm}$ to $1.4 \mathrm{~mm}$ of lateral plane from medial line were chosen according to stereotaxic atlas (Paxinos and Watson 2013). Sections were incubated in $80 \%$ formic acid (Sigma-Aldrich, Germany) for $40 \mathrm{~s}$ and heat pre-treated for antigen unmasking using antigen unmasking solution (Cat. H-3300, Vector Laboratories, USA) in autoclave 2100 Retriever (Aptum Biologics, UK), followed by incubation in $1 \% \mathrm{H}_{2} \mathrm{O}_{2}$ for $20 \mathrm{~min}$ and blocking using Section Block (Aptum Biologics, UK) for $30 \mathrm{~min}$. Sections were subsequently incubated overnight with primary antibody AT8 (1:1000; Thermo Fisher Scientific, Cat.MN1020, RRID (Research Resource Identifier): AB_223647) at $4^{\circ} \mathrm{C}$ recognizing phosphorylated tau protein (Ser-202 and Thr-205). The sections were incubated with biotinylated secondary antibody (Vectastain Elite ABC kit, Cat. PK-6102, Vector Laboratories, USA) at room temperature for $1 \mathrm{~h}$ and reacted with avidinbiotin-peroxidase complex for $1 \mathrm{~h}$. The immunoreaction was visualized with Vectastain VIP kit (Cat. SK-4600, Vector Laboratories, USA) without counterstaining. Microscopic analysis was performed using Olympus BX 51.

\section{Intraperitoneal glucose tolerance test and determination of metabolic parameters in plasma}

Intraperitoneal glucose tolerance test (IPGTT) was performed as follows: Two days before the termination of experiment the rats from both groups were injected intraperitoneally with a freshly prepared glucose load of $2 \mathrm{~g} / \mathrm{kg}$ of body weight (b.w.). A drop of blood was collected from tail immediately before glucose injection ( $0 \mathrm{~min}$ ) and 30, 60, 90 and $120 \mathrm{~min}$ after the injection for estimation of blood glucose using glucometer (Accu-Check Active, Roche Diagnostics, Germany). The rats fasted for $16 \mathrm{~h}$ before undergoing IPGTT. Identically the rats 
were starved overnight before sacrificing by decapitation and the blood was collected for plasma preparation using $\mathrm{K}_{3}$ EDTA tubes. Collected blood was centrifuged at $2000 \times g$ for $10 \mathrm{~min}$ at $4^{\circ} \mathrm{C}$ and aspirated plasma was stored at $-20^{\circ} \mathrm{C}$ prior analysis. After opening the abdominal cavity, the epididymal and retroperitoneal adipose tissue were removed and weighed. Adiposity index was calculated as a ratio of the weights of adipose tissues and corresponding weight of animal body. Plasma glucose and lipids were measured at external facility for the analysis of clinical samples (Synlab, Bratislava, Slovakia) using commercially available kits (Roche Molecular Diagnostics, Pleasanton, USA) assayed in COBAS Integra 800 multi-analyser (Roche, Switzerland). Insulin level was assayed by rat insulin RIA kit (Sigma-Aldrich, USA, Cat.No. RI-13K) and leptin levels were assayed by rat leptin ELISA kit (Millipore, USA, Cat.No. EZRL$83 \mathrm{~K}$ ) following the manufacturer's protocol, respectively.

\section{Real-time PCR profiling of leptin mRNA level}

Retroperitoneal white adipose tissues were after excision and weighing rapidly frozen in liquid nitrogen and stored at $-70^{\circ} \mathrm{C}$ until assayed. Total RNA was isolated from $100 \mathrm{mg}$ of frozen tissue using RNeasy Lipid Tissue Mini Kit (Qiagen, Cat. 74804, Hilden, Germany), according to the manufacturers protocol. $2 \mu \mathrm{g}$ of total RNA were reversely transcribed using Maxima First Strand cDNA Synthesis Kit (Thermo Fisher Scientific, Cat. K1642, Waltham, MA, USA). Real-time qPCR was performed using SYBR Green PCR Master Mix (Thermo Fisher Scientific, Cat. 4309155, Waltham, MA, USA) and 300 $\mathrm{nM}$ of each primer for leptin gene, $5^{\prime}$-CCAGGATGACACCAAAACCCTC-3' (sense) and 5'ATCCAGGCTCTCTGGCTTCTGC -3' (antisense). The relative amount of the leptin transcript was normalised to expression of the endogenous reference gene ribosomal protein S29 (Rps29), 5'-GCTGAACATGTGCCGACAGT-3' (sense) and 5'-GGTCGCTTAGTCCAACTTAATGAA-3' (antisense). The initial step in the reaction was $95^{\circ} \mathrm{C}$ for $10 \mathrm{~min}$, followed by 40 cycles of $95^{\circ} \mathrm{C}$ for $15 \mathrm{~s}$ and $60^{\circ} \mathrm{C}$ for $30 \mathrm{~s}$ for both primers. Reaction without cDNA as a template was used as a negative control. The qPCR was performed on ABI 7900HT thermal cycler (Applied Biosystems, Thermo Fisher Scientific, Waltham, MA, USA).

\section{Statistical analysis}

Graphpad Prism (version 6.07 for Windows; Graph Pad Software, USA) was used to carry out the statistical analysis. Assessment of the normality (D'Agostino \& Pearson omnibus normality test) of the data and ROUT test for outliers were performed, however, no data points were excluded from analysis. Due to the relatively limited sample size our study should be considered as exploratory. Differences between measures in WT and TG groups were analysed with unpaired Mann Whitney test or two-way ANOVA for IPGTT repeated measurements, respectively. Data are displayed as mean with 95\% confidence interval (CI). Results were considered to be statistically significant if $p<0.05$.

\section{Results}

Tau pathology is associated with reduction of body weight and downregulation of peripheral leptin

In this study we characterized the lipid and glucose metabolism disturbances under the neurodegenerative conditions on hypertensive genetic background. We employed SHR tau-transgenic animals developing the complete neuropathological signature of human tauopathy. Metabolic analysis of transgenic animals included determination of food consumption, body weight measurement, adiposity index, determination of glucose, insulin, leptin and lipid profile in the blood complemented with glucose tolerance test.

The analysed cohort consisted of pre-symptomatic transgenic animals (SHR72-TG, further referred as TG) that did not manifest any significant phenotypic signs of neurodegeneration; however, they already showed molecular and histopathological markers of neurofibrillary degeneration in comparison to age-matched wild type controls (SHR-WT, further as WT). TG animals have significantly reduced mean b.w. by $11 \%$ at six months of age when compared to controls (TG: $306 \mathrm{~g} v s$. WT: $344 \mathrm{~g}, p=0.0008$ ) (Fig. 1A). This reduction is also mirrored by the decreased adiposity index calculated from both, epididymal and retroperitoneal adipose tissue weights in TG animals compared to WT controls (TG: 1.3 vs. WT: $1.6, p=0.0036$ ) (Fig. 1B). However, daily food consumption did not differ between the TG and WT rats (TG: $5.62 \mathrm{~g} / 100 \mathrm{~g}$ of b.w. vs. WT: $5.6 \mathrm{~g} / 100 \mathrm{~g}$ of b.w., $p=0.5541$ ) (Fig. 1C), what represents a daily caloric intake of 18.13 $\mathrm{cal} / 100 \mathrm{~g}$ of b.w. in TG animals and $18.07 \mathrm{cal} / 100 \mathrm{~g}$ of b.w. in WT animals, respectively. The level of plasma leptin decreased significantly in transgenic animals (53\% of WT, TG: $0.9 \mathrm{ng} / \mathrm{ml} v s$. WT: $1.7 \mathrm{ng} / \mathrm{ml}, p=0.0002$ ) (Fig. 1D). The effect was evident already at the level of leptin mRNA expression in retroperitoneal fat tissue, which was reduced in TG rats to a similar extent (51\% of WT, TG: 0.47 -fold $v s$. WT: 0.93 -fold, $p=0.0003$ ) (Fig. 1E). TG animals showed unaltered plasma lipid parameters of total cholesterol (TChol), low density lipoproteins (LDL), triglycerides (TGL) and very low density lipoproteins (VLDL), but significantly reduced high density lipoproteins (HDL, 88\% of WT, TG: $0.94 \mathrm{mmol} / \mathrm{l} v s$. WT: 1.07 $\mathrm{mmol} / \mathrm{l}, p=0.0226$ ) (Fig. $1 \mathrm{~F}$ ). Furthermore, to determine the changes in metabolism of glucose and insulin due to the pathological tau, we measured these parameters in plasma. The analysis revealed no difference in levels of glucose (TG: $4.6 \mathrm{mmol} / \mathrm{l} v s$. WT: $5.0 \mathrm{mmol} / \mathrm{l}, p=0.0988$ ) and insulin (TG: $0.5 \mathrm{ng} / \mathrm{ml} v s$. WT: $0.56 \mathrm{ng} / \mathrm{ml}, p=0.5907)$ between TG and 
WT animals (Fig. 1G,H). IPGTT did not show any significant difference between tauopathic and control rats (Fig. 1I).

Hypothalamic and brainstem nuclei targeted by leptin are free of tau pathology

To test the hypothesis that decrease of plasma leptin and its mRNA expression in fat tissue is related to the formation of tau pathology in the brain we analysed specific leptin-responsive nuclei, ARC, VMH, DMH and PVH, that are localized in hypothalamus and NTS localized in brainstem. The staining with AT8 antibody that recognizes early to late stages of neurofibrillary degeneration identified tau protein aggregates in the brainstem of TG rats, resembling a typical picture of tau pathology in SHR72 model. Parallel staining of control brain of WT rats did not show any signs of tau pathology (Fig. 2A,B). However, detailed immunohistochemical staining of the brain regions regulated by leptin revealed that there is no
A

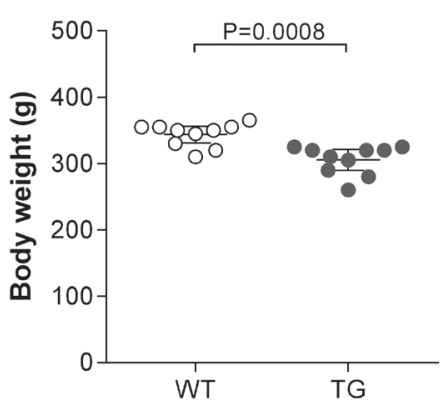

D

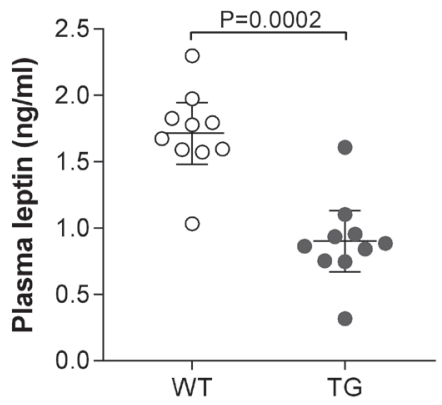

G

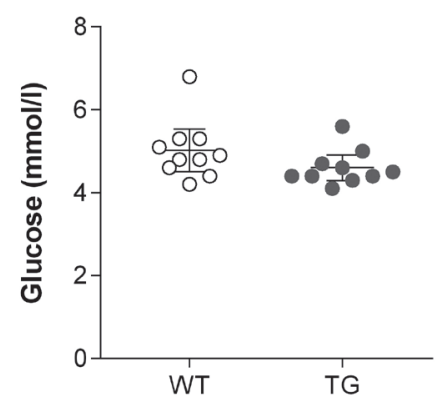

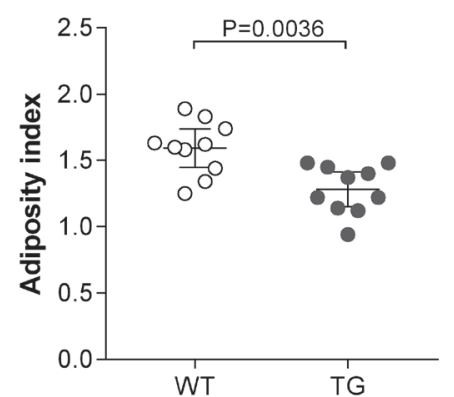

E

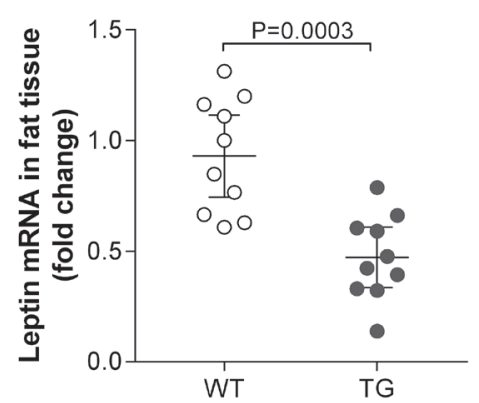

H

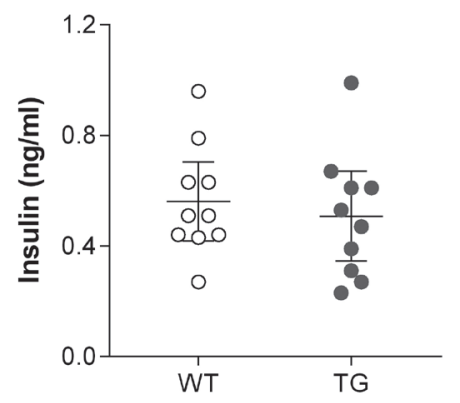

C

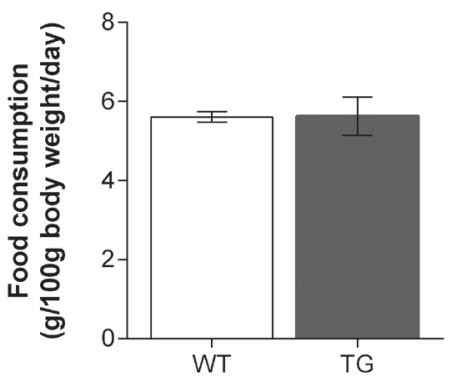

$\mathbf{F}$

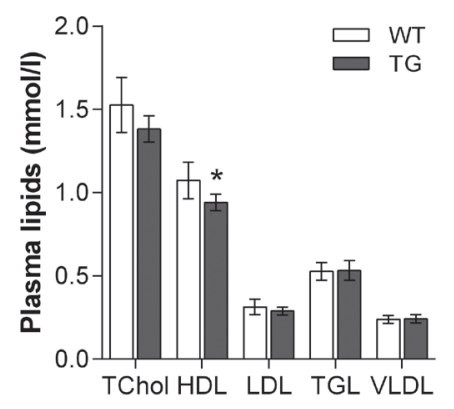

I

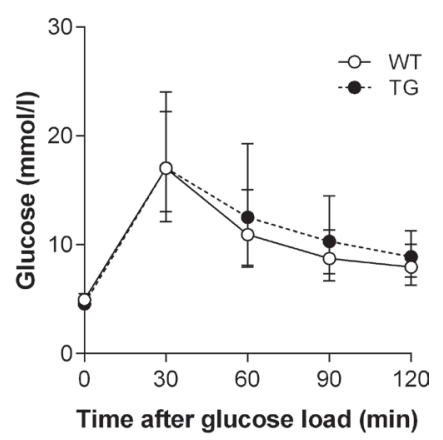

Figure 1. Differences in body weight and metabolic parameters in SHR72 transgenic rats. A. Comparative analysis revealed significant loss of body weight of transgenic (TG) rats when compared to age-matched wild type (WT) controls. B. This deficit is also reflected as a drop of adiposity index in TG animals, but is (C) not related to different food consumption. D. Decreased level of plasma leptin in TG rats as confirmed by the lower leptin expression (E) in retroperitoneal fat tissue. F. Profile of plasma lipids showed decreased level of HDL in TG animals. Level of $(\mathbf{G})$ glucose and $(\mathbf{H})$ insulin is not affected by overnight fasting. I. Intraperitoneal glucose tolerance test revealed no difference between TG $(n=10)$ and WT $(n=10)$ animals (data are displayed as mean with $95 \%$ confidence interval; ${ }^{*} p<$ 0.05 vs. WT). TChol, total cholesterol; HDL, high density lipoproteins. 


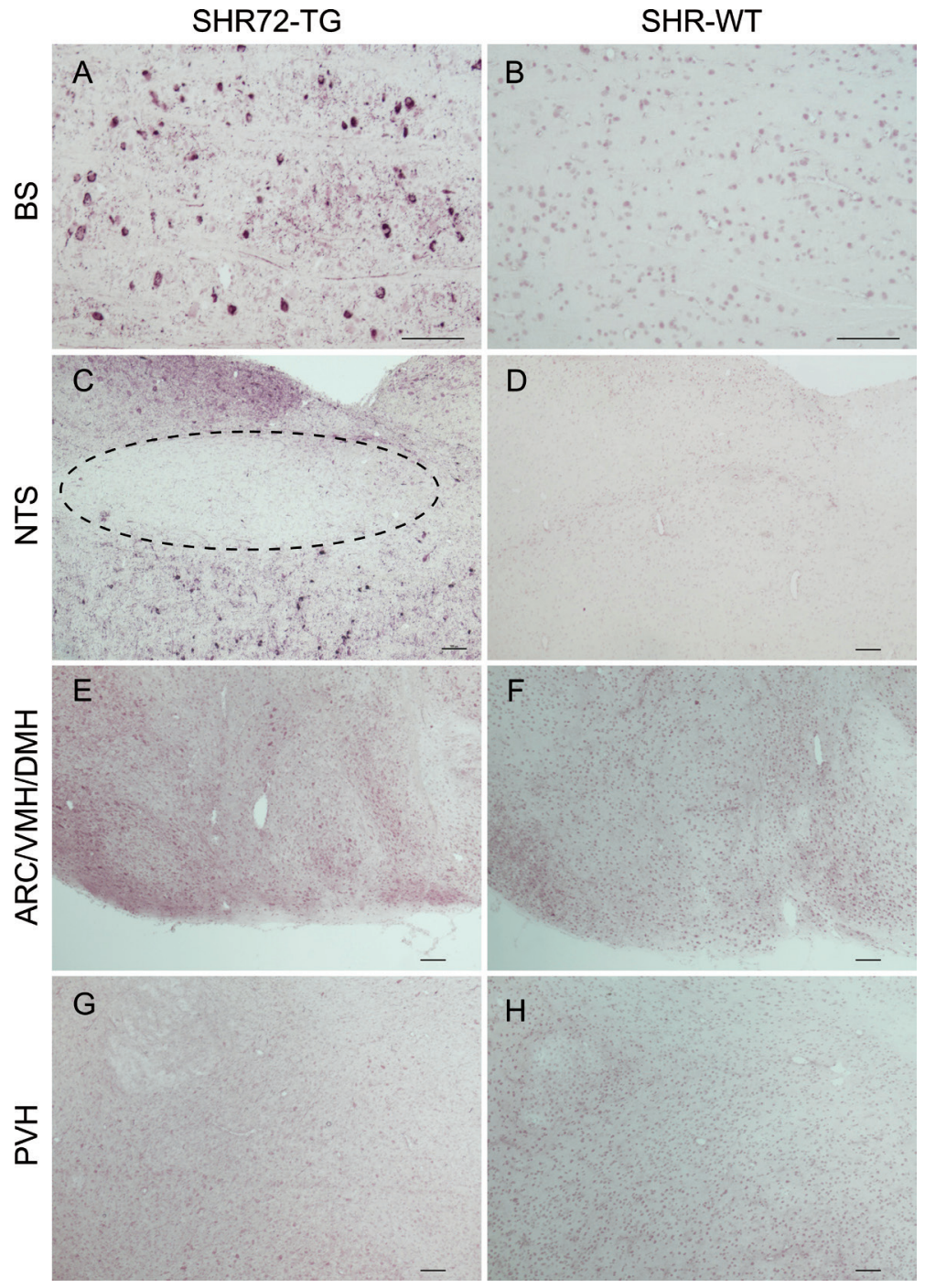

Figure 2. Hypothalamic and brainstem nuclei targeted by leptin are free of tau pathology. A. Neurofibrillary pathology in transgenic SHR72 rats is localized in brainstem (BS) while the (B) control WT rats show negative staining with AT8 antibody. C.,D. The brainstem nuclei of nucleus tractus solitarius (NTS - area marked by dashed line) and hypothalamic area of (E,F) arcuate nucleus (ARC) including ventromedial nucleus $(\mathrm{VMH})$ and dorsomedial nucleus $(\mathrm{DMH})$ together with $(\mathbf{G}, \mathbf{H})$ paraventricular nucleus (PVH) regulated by leptin are free of tau protein phosphorylation or neurofibrillary tangles as stained by AT8 antibody. Scale bar $=100 \mu \mathrm{m}$. AT8 antibody is specific for tau protein phosphorylated at Ser202/Ser205. tau pathology present in area of ARC or NTS. Detection of neurofibrillary tangles and neuropil threads with AT8 in ARC, $\mathrm{VMH}, \mathrm{DMH}, \mathrm{PVH}$ and NTS areas was also negative showing only background staining (Fig. 2C-H). These data indicate that downregulation of peripheral leptin is not directly caused by the formation of tau protein aggregates, since the leptin responsive brain regions are free of any signs of tau pathology.

\section{Discussion}

Neurodegeneration in patients with $\mathrm{AD}$ and other neuropathies is associated with peripheral changes including metabolic disturbances, hypertension, alterations of body weight and fat metabolism (Lee 2011; McGuire and Ishii 2016; Mogi 2019; den Brok et al. 2021; Pierzchlinska et al. 2021; Ungvari et al. 2021). These disorders are generally considered risk factors for the development of neurodegeneration, but it is difficult to study in humans whether or not they may also be the consequences of neuropathology. This can be clearly demonstrated using a suitable experimental animal model.

In our previous work with normotensive rats (WKY72) we have partially answered this question and confirmed the metabolic disturbances as a consequence of neurodegeneration. We have shown that expression of truncated tau protein in WKY72 rats induces downregulation of peripheral leptin, alteration of lipid profile and disturbances in glucose metabolism. Moreover, peripheral level of leptin in animals was negatively correlated with degree of neurofibrillary degeneration in the brain (Cente et al. 2020).

We have now investigated the effect of experimentally induced tau protein neurodegeneration on lipid and glucose metabolism in chronic hypertension, using spontaneously hypertensive rats (strain SHR72). 
We have also focused on the detailed determination of tau protein neuropathology (mainly the presence of neurofibrillary tangles) in the brainstem areas, which are involved in maintaining energy homeostasis and physiological levels of peripheral leptin. Specifically, we examined the leptin receptors-containing brain regions such as, $\mathrm{ARC}, \mathrm{VMH}, \mathrm{DMH}$ and $\mathrm{PVH}$ that are localized in hypothalamus and NTS in brainstem (Myers et al. 2009; Sutton et al. 2016) aiming to determine whether the tau pathology eventually present in these areas of central nervous system might affect the "brain-periphery" leptin signalling.

Based on the analyses of the hypertensive animals and comparison to normotensive ones we can conclude that effect of experimentally induced neurodegeneration is very similar in both animal strains although the neurodegeneration in hypertensive animals leads to more profound decrease of peripheral leptin.

The data on decreased body weight and adiposity index in transgenic animals are in line with observations in $\mathrm{AD}$ patients, where lowered body weight, sarcopenia, and reduced fat mass in comparison to healthy controls were reported (Guerin et al. 2005; Theodoropoulou et al. 2012; Gratuze et al. 2018). We did not quantify parameters related to sarcopenia in this particular experiment. However, we have previously reported the atrophy of hind leg musculature at the age of 7 month, together with loss of the muscular strength and agility during aging, suggesting signs of sarcopenia at later stages of neurofibrillary degeneration in transgenic rats (Korenova et al. 2009). Interestingly, in our model we have observed highly significant weight loss accompanied by a loss of adipose tissue mass, while the food consumption remained unchanged. This might point to hypermetabolism in transgenic animals, which is known from humans and was also observed in transgenic mouse models of AD (Knight et al. 2012; Zheng et al. 2018).

Although, majority of lipid parameters determined in plasma of tauopathic animals is at physiological level, mild reduction of HDL cholesterol in plasma of TG animals was detected. Several studies indicate that higher risk of AD is associated with low levels of HDL cholesterol (Reitz et al. 2010; Warren et al. 2012; Tang et al. 2019). Our findings suggest that HDL related dyslipidaemia might be adverse concomitant metabolic disturbance of the progressing tau protein-induced neurodegeneration and support the view that normalized or higher HDL may reduce the risk of $\mathrm{AD}$ and related tauopathies. Recent evidence from human studies and animal models supports the hypothesis that HDL cholesterol protects against cerebrovascular dysfunction in AD (Button et al. 2019).

Circulating leptin concentrations are proportional to adipose tissue mass (Stern et al. 2016). Accordingly, in our study the lower b.w. and adipose tissue mass in TG animals were accompanied by a decrease in adipose leptin transcription (mRNA) and plasma leptin levels compared to wild type rats. Leptin was described as a neuroprotective molecule and potential cognitive enhancer as it rapidly alters glutamate receptor trafficking processes and in turn the efficacy of hippocampal excitatory synaptic transmission (Mejido et al. 2020). Furthermore, it was demonstrated that lower serum leptin in individuals with mild cognitive impairment is associated with lower hippocampal volume suggesting that inefficient leptin signaling could partly contribute to decreases in memory performance (Witte et al. 2016). Akin to this study higher circulating leptin was associated with a reduced incidence of dementia and $\mathrm{AD}$ in non-obese individuals (Lieb et al. 2009). Accumulating evidence proposed leptin as a potentially important therapeutic target for treatment of neurodegenerative conditions. Chronic administration of leptin to transgenic mouse model of $\mathrm{AD}$ resulted in attenuation of amyloid betainduced neurodegeneration and reduction of senile plaque pathology in the brain (Fewlass et al. 2004; Calio et al. 2021). These results support the assumption that peripheral levels of leptin are strongly associated with degree of neurodegeneration as they also inversely correlate with amount of insoluble tau protein present in neurofibrillary tangles (Cente et al. 2020). As the metabolic regulation involves complex interplay of many processes, it is likely that the risk for developing neurodegenerative disorder is affected by a range of other leptin-unrelated confounding factors including gender, diet, exercise and age.

The relationship of $\mathrm{AD}$ and glucose metabolism disorders is complex and remains poorly understood at the molecular level. Diabetes is implicated as a risk factor for development of $\mathrm{AD}$ since these two diseases share some common pathophysiological features (Hanson and Rubinow 2021). Moreover, diabetes with its vascular complications may result in neurodegenerative disease (Bosco et al. 2011; van der Flier et al. 2018; Kubis-Kubiak et al. 2019). However, in our model of induced tauopathy no signs of peripheral insulin resistance were noticed. Glucose utilization of TG animals as well as fasting glucose and insulin concentrations were not different from those of controls. We conclude that tauopathy itself does not affect insulin sensitivity in hypertensive SHR72 rats, in contrast to the normotensive model WKY72, where we found reduced fasting glucose and insulin concentrations in TG animals (Cente et al. 2020).

To explain the deleterious effect of neurofibrillary degeneration on peripheral leptin we examined neuropathology in leptin-responsive areas of hypothalamus and brainstem. Using a histopathological marker for clinical diagnostics of tauopathy, the AT8 antibody, we investigated the presence of tau pathology in specific brain nuclei involved in the leptin projection circuits. Interestingly, our data indicate that major leptin-responsive regions of ARC, PVH and NTS in the brain of tauopathic animals do not exhibit any signs of tau proteininduced neurodegeneration. Since the tauopathic animals have normal food intake despite lower peripheral leptin and the leptin projection circuit involving ARC, PVH and NTS is free of any signs of tau pathology it seems that the neurons responsive to energy-related signals in these areas still mediate the peripheral satiety signals with in a physiological 
manner. However, we cannot completely exclude that other leptin-regulated circuits in the brain are affected by the tau pathology present in adjacent brainstem areas connected to the ARC, PVH or NTS nuclei inhibiting the leptin signaling within the central nervous system of tauopathic animals.

In conclusion, our data revealed that truncated tau protein-induced neurodegeneration at the hypertensive genetic background is associated with downregulation of peripheral leptin, reduction of b.w., adiposity index and decrease of HDL cholesterol underlying the link between neurodegeneration in the brain and altered fat metabolism in periphery as observed in $\mathrm{AD}$ and other tauopathies. The decrease in leptin concentration in plasma can lead to accelerated neurodegeneration, since its neuroprotective function is attenuated. However, the molecular nature of direct or indirect connection between pathological tau and peripheral leptin deficiency remains to be elucidated by further research. The experimental model we used in our study can be employed as a model of choice for detailed clarifying the molecular signaling underlying the neurodegenerationdriven disturbance of peripheral lipid metabolism.

Author contributions. Investigation, TS, KK, LB; data curation, MC, LF, NPI; writing-original draft preparation, MC, PF; writingreview and editing, SZ, RS; funding acquisition, PF, MC, SZ. All authors have read and approved the final version of the manuscript.

Funding. This research was co-funded by Slovak Research and Development Agency, grant number APVV-18-0515, APVV-200421, APVV-17-0668 and Ministry of Education, Science, Research and Sport of the Slovak Republic, in the form of research grants VEGA 2/0160/20, 2/0154/19 and 2/0118/19.

Compliance with Ethical Standards. All animals used in experiments were obtained from animal facility of NIU SAS (Institute of Neuroimmunology, Slovak Academy of Sciences, Bratislava, Slovakia) that is approved by the State Veterinary and Food Administration of the Slovak Republic (approval SK CH 12016). The study was conducted according to the Slovak and European Community Guidelines, with the approval of the Institute's Ethical Committee and State Veterinary and Food Administration of the Slovak Republic (No.4429-/16-221k, approved on January 9, 2017).

Conflicts of interest. The authors declare no conflict of interest. The funders had no role in the design of the study; in the collection, analyses, or interpretation of data; in the writing of the manuscript, or in the decision to publish the results.

\section{References}

Abd Alkhaleq H, Kornowski R, Waldman M, Levy E, Zemel R, Nudelman V, Shainberg A, Miskin R, Hochhauser E (2020): Leptin modulates gene expression in the heart and cardiomyocytes towards mitigating ischemia-induced damage. Exp. Cell Res. 397, 112373 https://doi.org/10.1016/j.yexcr.2020.112373

Beltowski J (2006): Role of leptin in blood pressure regulation and arterial hypertension. J. Hypertens. 24, 789-801 https://doi.org/10.1097/01.hjh.0000222743.06584.66

Bosco D, Fava A, Plastino M, Montalcini T, Pujia A (2011): Possible implications of insulin resistance and glucose metabolism in Alzheimer's disease pathogenesis. J. Cell Mol. Med. 15, 18071821 https://doi.org/10.1111/j.1582-4934.2011.01318.x

Button EB, Robert J, Caffrey TM, Fan J, Zhao W, Wellington CL (2019): HDL from an Alzheimer's disease perspective. Curr. Opin. Lipidol. 30, 224-234 https://doi.org/10.1097/MOL.0000000000000604

Calio ML, Mosini AC, Marinho DS, Salles GN, Massinhani FH, Ko GM, Porcionatto MA (2021): Leptin enhances adult neurogenesis and reduces pathological features in a transgenic mouse model of Alzheimer's disease. Neurobiol. Dis. 148, 105219 https://doi.org/10.1016/j.nbd.2020.105219

Cente M, Zorad S, Smolek T, Fialova L, Paulenka Ivanovova N, Krskova K, Balazova L, Skrabana R, Filipcik P (2020): Plasma leptin reflects progression of neurofibrillary pathology in animal model of tauopathy. Cell. Mol. Neurobiol. (in press) https://doi.org/10.1007/s10571-020-00972-6

den Brok M, van Dalen JW, Abdulrahman H, Larson EB, van Middelaar T, van Gool WA, van Charante EPM, Richard E (2021): Antihypertensive medication classes and the risk of dementia: a systematic review and network meta-analysis. J. Am. Med. Dir. Assoc. 22, 1386-1395 https://doi.org/10.1016/j.jamda.2020.12.019

Emmerzaal TL, Kiliaan AJ, Gustafson DR (2015): 2003-2013: a decade of body mass index, Alzheimer's disease, and dementia. J. Alzheimers. Dis. 43, 739-755 https://doi.org/10.3233/JAD-141086

Fewlass DC, Noboa K, Pi-Sunyer FX, Johnston JM, Yan SD, Tezapsidis N (2004): Obesity-related leptin regulates Alzheimer's Abeta. FASEB J. 18, 1870-1878 https://doi.org/10.1096/fj.04-2572com

Gratuze M, Joly-Amado A, Vieau D, Buee L, Blum D (2018): Mutual relationship between tau and central insulin signalling: consequences for AD and Tauopathies? Neuroendocrinology 107, 181-195 https://doi.org/10.1159/000487641

Guerin O, Andrieu S, Schneider SM, Milano M, Boulahssass R, Brocker P, Vellas B (2005): Different modes of weight loss in Alzheimer disease: a prospective study of 395 patients. Am. J. Clin. Nutr. 82, 435-441 https://doi.org/10.1093/ajcn/82.2.435

Hanson AJ, Rubinow KB (2021): Optimizing clinical phenotyping to better delineate the complex relationship between type 2 diabetes and Alzheimer's disease. Clin. Transl. Sci. 14, 1681-1688 https://doi.org/10.1111/cts.13024

Knight EM, Verkhratsky A, Luckman SM, Allan SM, Lawrence CB (2012): Hypermetabolism in a triple-transgenic mouse model of Alzheimer's disease. Neurobiol. Aging 33, 187-193 https://doi.org/10.1016/j.neurobiolaging.2010.02.003

Korenova M, Zilka N, Stozicka Z, Bugos O, Vanicky I, Novak M (2009): NeuroScale, the battery of behavioral tests with novel scoring system for phenotyping of transgenic rat model of tauopathy. J. Neurosci. Methods 177, 108-114 
https://doi.org/10.1016/j.jneumeth.2008.09.027

Koson P, Zilka N, Kovac A, Kovacech B, Korenova M, Filipcik P, Novak M (2008): Truncated tau expression levels determine life span of a rat model of tauopathy without causing neuronal loss or correlating with terminal neurofibrillary tangle load. Eur. J. Neurosci. 28, 239-246 https://doi.org/10.1111/j.1460-9568.2008.06329.x

Kubis-Kubiak AM, Rorbach-Dolata A, Piwowar A (2019): Crucial players in Alzheimer's disease and diabetes mellitus: Friends or foes? Mech. Ageing Dev. 181, 7-21 https://doi.org/10.1016/j.mad.2019.03.008

Lee EB (2011): Obesity, leptin, and Alzheimer's disease. Ann. NY Acad. Sci. 1243, 15-29 https://doi.org/10.1111/j.1749-6632.2011.06274.x

Lieb W, Beiser AS, Vasan RS, Tan ZS, Au R, Harris TB, Roubenoff R, Auerbach S, DeCarli C, Wolf PA et al. (2009): Association of plasma leptin levels with incident Alzheimer disease and MRI measures of brain aging. JAMA 302, 2565-2572 https://doi.org/10.1001/jama.2009.1836

McGuire MJ, Ishii M (2016): Leptin dysfunction and Alzheimer's disease: Evidence from cellular, animal, and human studies. Cell Mol. Neurobiol. 36, 203-217 https://doi.org/10.1007/s10571-015-0282-7

Mejido DCP, Peny JA, Vieira MNN, Ferreira ST, De Felice FG (2020): Insulin and leptin as potential cognitive enhancers in metabolic disorders and Alzheimer's disease. Neuropharmacology 171, 108115 https://doi.org/10.1016/j.neuropharm.2020.108115

Mogi M (2019): Could management of blood pressure prevent dementia in the elderly? Clin. Hypertens. 25, 27 https://doi.org/10.1186/s40885-019-0135-7

Myers MG Jr, Munzberg H, Leinninger GM, Leshan RL (2009): The geometry of leptin action in the brain: more complicated than a simple ARC. Cell Metab. 9, 117-123 https://doi.org/10.1016/j.cmet.2008.12.001

Pantsulaia I, Trofimova S, Kobyliansky E, Livshits G (2009): Relationship between obesity, adipocytokines, and blood pressure: possible common genetic and environmental factors. Am. J. Hum. Biol. 21, 84-90 https://doi.org/10.1002/ajhb.20821

Paxinos G, Watson C (2013): The Rat Brain in Stereotaxic Coordinates. 7th edition. Academic Press. eBook ISBN: 9780124157521

Pierzchlinska A, Kwasniak-Butowska M, Slawek J, Drozdzik M, Bialecka M (2021): Arterial blood pressure variability and other vascular factors contribution to the cognitive decline in Parkinson's disease. Molecules 26, 1526 https://doi.org/10.3390/molecules26061523

Reitz C, Tang MX, Schupf N, Manly JJ, Mayeux R, Luchsinger JA (2010): Association of higher levels of high-density lipoprotein cholesterol in elderly individuals and lower risk of late-onset Alzheimer disease. Arch. Neurol. 67, 1491-1497 https://doi.org/10.1001/archneurol.2010.297

Simonds SE, Pryor JT, Cowley MA (2017): Does leptin cause an increase in blood pressure in animals and humans? Curr. Opin. Nephrol. Hypertens 26, 20-25 https://doi.org/10.1097/MNH.0000000000000287
Stern JH, Rutkowski JM, Scherer PE (2016): Adiponectin, leptin, and fatty acids in the maintenance of metabolic homeostasis through adipose tissue crosstalk. Cell Metab. 23, 770-784 https://doi.org/10.1016/j.cmet.2016.04.011

Stozicka Z, Zilka N, Novak P, Kovacech B, Bugos O, Novak M (2010): Genetic background modifies neurodegeneration and neuroinflammation driven by misfolded human tau protein in rat model of tauopathy: implication for immunomodulatory approach to Alzheimer's disease. J. Neuroinflammation 7, 64 https://doi.org/10.1186/1742-2094-7-64

Sutton AK, Myers MG Jr, Olson DP (2016): The role of PVH circuits in leptin action and energy balance. Annu. Rev. Physiol. 78, 207-221 https://doi.org/10.1146/annurev-physiol-021115-105347

Tang Q, Wang F, Yang J, Peng H, Li Y, Li B, Wang S (2019): Revealing a novel landscape of the association between blood lipid levels and Alzheimer's disease: A meta-analysis of a case-control study. Front. Aging Neurosci. 11, 370 https://doi.org/10.3389/fnagi.2019.00370

Theodoropoulou A, Metallinos IC, Psyrogiannis A, Vagenakis GA, Kyriazopoulou V (2012): Ghrelin and leptin secretion in patients with moderate Alzheimer's disease. J. Nutr. Health Aging 16, 472-477

https://doi.org/10.1007/s12603-012-0058-4

Ungvari Z, Toth P, Tarantini S, Prodan CI, Sorond F, Merkely B, Csiszar A (2021): Hypertension-induced cognitive impairment: from pathophysiology to public health. Nat. Rev. Nephrol. 17, 639-654 https://doi.org/10.1038/s41581-021-00430-6

van der Flier WM, Skoog I, Schneider JA, Pantoni L, Mok V, Chen CLH, Scheltens P (2018): Vascular cognitive impairment. Nat. Rev. Dis. Primers 4, 18003 https://doi.org/10.1038/nrdp.2018.3

Wang H, Necheles J, Birne JS, Li Z, Xing H, Tang G, Christoffel KK, Brickman WJ, Zimmerman D, Wang X (2012): Association of adipokines with blood pressure in rural Chinese adolescents. J. Hum. Hypertens. 26, 493-501 https://doi.org/10.1038/jhh.2011.64

Warren MW, Hynan LS, Weiner MF (2012): Lipids and adipokines as risk factors for Alzheimer's disease. J. Alzheimers Dis. 29, 151-157 https://doi.org/10.3233/JAD-2012-111385

Witte AV, Kobe T, Graunke A, Schuchardt JP, Hahn A, Tesky VA, Pantel J, Floel A (2016): Impact of leptin on memory function and hippocampal structure in mild cognitive impairment. Hum. Brain Mapp. 37, 4539-4549

https://doi.org/10.1002/hbm.23327

Zheng H, Zhou Q, Du Y, Li C, Xu P, Lin L, Xiao J, Gao H (2018): The hypothalamus as the primary brain region of metabolic abnormalities in APP/PS1 transgenic mouse model of Alzheimer's disease. Biochim. Biophys. Acta Mol. Basis Dis. 1864, 263-273 https://doi.org/10.1016/j.bbadis.2017.10.028

Zilka N, Filipcik P, Koson P, Fialova L, Skrabana R, Zilkova M, Rolkova G, Kontsekova E, Novak M (2006): Truncated tau from sporadic Alzheimer's disease suffices to drive neurofibrillary degeneration in vivo. FEBS Lett. 580, 3582-3588 https://doi.org/10.1016/j.febslet.2006.05.029

Received: June 4, 2021

Final version accepted: September 20, 2021 